\title{
A Novel Interdigital Capacitor Pressure Sensor Based on LTCC Technology
}

\author{
Qiulin Tan, ${ }^{1,2,3}$ Mingliang Yang, ${ }^{1,2}$ Tao Luo, ${ }^{2}$ Wei Liu, ${ }^{1}$ Chao Li, ${ }^{1}$ \\ Chenyang Xue, ${ }^{2}$ Jun Liu, ${ }^{1,2}$ Wendong Zhang, ${ }^{1}$ and Jijun Xiong ${ }^{1,2}$ \\ ${ }^{1}$ Key Laboratory of Instrumentation Science \& Dynamic Measurement, North University of China, \\ Ministry of Education, Tai Yuan 030051, China \\ ${ }^{2}$ Science and Technology on Electronic Test \& Measurement Laboratory, North University of China, Tai Yuan 030051, China \\ ${ }^{3}$ National Key Laboratory of Fundamental Science of Micro/Nano-Device and System Technology, \\ Chongqing University, Chongqing 400044, China
}

Correspondence should be addressed to Qiulin Tan; tanqiulin.99@163.com and Jijun Xiong; xiongjijun@nuc.edu.cn

Received 22 February 2014; Revised 8 June 2014; Accepted 8 June 2014; Published 23 June 2014

Academic Editor: Xinyong Dong

Copyright (C) 2014 Qiulin Tan et al. This is an open access article distributed under the Creative Commons Attribution License, which permits unrestricted use, distribution, and reproduction in any medium, provided the original work is properly cited.

A novel passive wireless pressure sensor is proposed based on LTCC (low temperature cofired ceramic) technology. The sensor employs a passive LC circuit, which is composed of a variable interdigital capacitor and a constant inductor. The inductor and capacitor were fabricated by screen-printing. Pressure measurement is tested using a wireless mutual inductance coupling method. The experimental sensitivity of the sensor is about $273.95 \mathrm{kHz} /$ bar below 2 bar. Experimental results show that the sensor can be read out wirelessly by external antenna at $600^{\circ} \mathrm{C}$. The max readout distance is $3 \mathrm{~cm}$ at room temperature. The sensors described can be applied for monitoring of gas pressure in harsh environments, such as environment with high temperature and chemical corrosion.

\section{Introduction}

Passive wireless sensor has characteristics of having no batteries and contactless signal readout and nonpollution, which make it have extensive application prospects in many fields, such as high-temperature measurement, hermetic space, and rotating components. Wireless inductor sensor is a new type of wireless sensor. In recent years, LTCC is widely used in applications like RF circuits, microwave device, package, and so forth, but they seldom have been used to fabricate capacitive pressure sensors. LTCC technology is the method which can make multilayer ceramic tapes and printed conductive metal figures a whole multilayer interconnection structure by sintering at $800 \sim 950^{\circ} \mathrm{C}$ temperature. Passivewireless pressure measurement in harsh environments such as high temperatures has become increasingly critical in automotive, aerospace, and industrial applications [1-3]. Most pressure sensors are made by micromachining silicon, and they have many advantages in terms of small size and fast response. However, the circuitry ultimately limits the operational temperatures to $<150^{\circ} \mathrm{C}$ [4-6]. Typical temperatures for high-temperature environments applications can range from 200 to $1000^{\circ} \mathrm{C}$, requiring the development of novel sensor. The proposed sensor in this paper which is fabricated in LTCC green tapes based on LC resonant principle is prospective to solve those problems.

This paper proposes a passive wireless sensor for remote pressure monitoring in high-temperature environment and also can be used for environment with hazardous gas. It consists of a fixed inductor and variable capacitor. The noncontact measurement technology is used to obtain pressure information $[7,8]$. The proposed pressure sensors are totally passive, which means that there are no batteries and the possibility for the occurrence of electric spark is eliminated, which is critical for the hazardous gas environment. The novel pressure sensor realized in LTCC technology can work at temperatures from 400 to $600^{\circ} \mathrm{C}$. It is expected to wirelessly measure pressure up to $600^{\circ} \mathrm{C}$ and last up to 30 minutes. 


\section{Design}

2.1. Principle of Measurement. The principle of measurement is based on a passive LC circuit. The sensor has two significant components, a planar spiral inductor and a variable interdigital capacitor which is a sensing element.

The resonant frequency of sensor can be retrieved from the well-known expression:

$$
f_{H}=\frac{1}{2 \pi \sqrt{L_{S} C_{S}}} .
$$

$L_{s}$ and $C_{s}$ are inductance and capacitance of the sensor. It is evident that any variation of capacitance or inductance values results in a shift of resonant frequency $f_{H}$ of the LC resonant circuit. The pressure sensor presented in this paper utilizes variation of external pressure to change capacitance values, which makes variation of sensor frequency. Therefore, the size of the external pressure can be got by the detection of resonant frequency change in the $\mathrm{LC}$ circuit.

Pressure measurement is tested using a wireless mutual inductance coupling method [9]. In order to obtain the frequency-pressure relationship, an Agilent E4991 impedance analyzer is used to test the sensors. The magnetic link between two inductively coupled coils allows the impedance analyzer to measure impedance variation of the device wirelessly as it is excited. The induction of antenna produces the alternating magnetic field. When the antenna is near the sensor, the magnetic energy is transmitted by the inductance coil of the LC circuit [10]. Antenna and sensor are contacted without wires. The power supply of sensor is electromagnetic wave energy. The antenna transmits energy to the sensor. It realizes the passive wireless detection. The wireless measurement way is presented in Figure 1.

The sensor resonant frequency can be obtained through testing antenna impedance phase with Agilent impedance analyzer. The total impedance of the reader antenna can be determined as

$$
Z=R_{a}+j 2 \pi 2 \pi_{a}\left[1+\frac{k^{2}\left(f / f_{0}\right)^{2}}{1+j(1 / Q)\left(f / f_{0}\right)-\left(f / f_{0}\right)^{2}}\right],
$$

where $R_{a}$ is the resistance of reader antenna, $L_{a}$ is inductance of reader antenna, and $Q$ is the quality factor of sensor. From (2), it is obvious that $Z$ relates to the sensor resonant frequency $f_{0}$, and $f_{0}$ can be obtained by measuring input impedance $Z$.

$k$ is the coupling coefficient between the sensor and the antenna and is given by

$$
k=\frac{M}{\sqrt{L_{s} L_{a}}} .
$$

$M$ is the mutual inductance.

2.2. Sensor Structure Design. Figure 2 is a cross-sectional view of the interdigital capacitor sensor. Inductance is a plane spiral inductor. Capacitor is an interdigital capacitor.

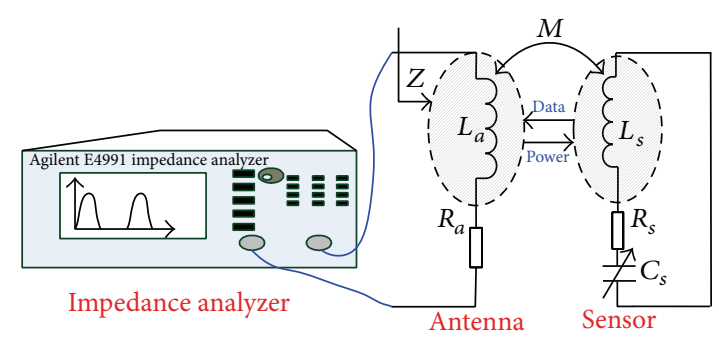

FIgURE 1: Schematic of test system circuit.

The sensor consists of four layers of LTCC ceramic tape. The first layer is sensitive pressure film of the sensor. Under external pressure, pressure sensitive membrane will produce deformation, causing the change of capacitance size and then the change of frequency. Inductor capacitor can be known from the following equation [11]:

$$
L_{s}=\frac{\mu n^{2} d_{\mathrm{avg}}}{2}\left[\ln \left(\frac{2.46}{\rho_{s}}\right)+0.2 \rho_{s}^{2}\right] .
$$

In formula (4), $\mu$ is vacuum magnetic conductivity. It is a constant value. The spiral number of turns is $n$; the equivalent spiral representations have inner, outer, and average diameters $d_{\mathrm{in}}, d_{\text {out }}$, and $d_{\text {avg }}$, respectively. $d_{\text {avg }}=d_{1}+$ $d_{2}+\cdots+d_{n} / n, \rho_{s}=d_{\text {out }}-d_{\text {in }} / d_{\text {out }}+d_{\text {in }}, \varepsilon_{r}$ is the relative dielectric constant of the LTCC green tape, and $\varepsilon_{0}$ is absolute dielectric constant in vacuum.

Interdigital capacitor can be known from the following equation [7]:

$$
C_{S}=L_{c}\left(N_{C}-1\right) \varepsilon_{0} \frac{1+\varepsilon_{r} K\left[\left(1-\left(g_{c} /\left(w_{c}+g_{c}\right)\right)^{2}\right)^{1 / 2}\right]}{2 K\left(g_{c} /\left(w_{c}+g_{c}\right)\right)} .
$$

In formula (3), $L_{c}$ is the length of the interdigital capacitor electrode. $N_{c}$ is the number of the interdigital capacitor electrodes. $g_{c}$ is the distance between one electrode and another in the interdigital capacitor. $w_{c}$ is the width of the interdigital capacitor electrode. Based on the principle above, this paper designed a passive wireless pressure sensor in which the resonant frequency is about $40.68 \mathrm{MHz}$.

According to Figure 2, $L_{c}$ increases with the increase in the external pressure. From formula (1) and formula (5), the frequency of the sensor decreases with the increase in the capacitance and also decreases with the increase in the external pressure.

\section{Fabrication}

LTCC is short for low temperature cofiring ceramic technology. The fabrication process is illustrated in Figure 2. The sensor consists of four layers of LTCC ceramic tape. The top ceramic tape creates the pressure sensitive diaphragm of the mechanical structure. 


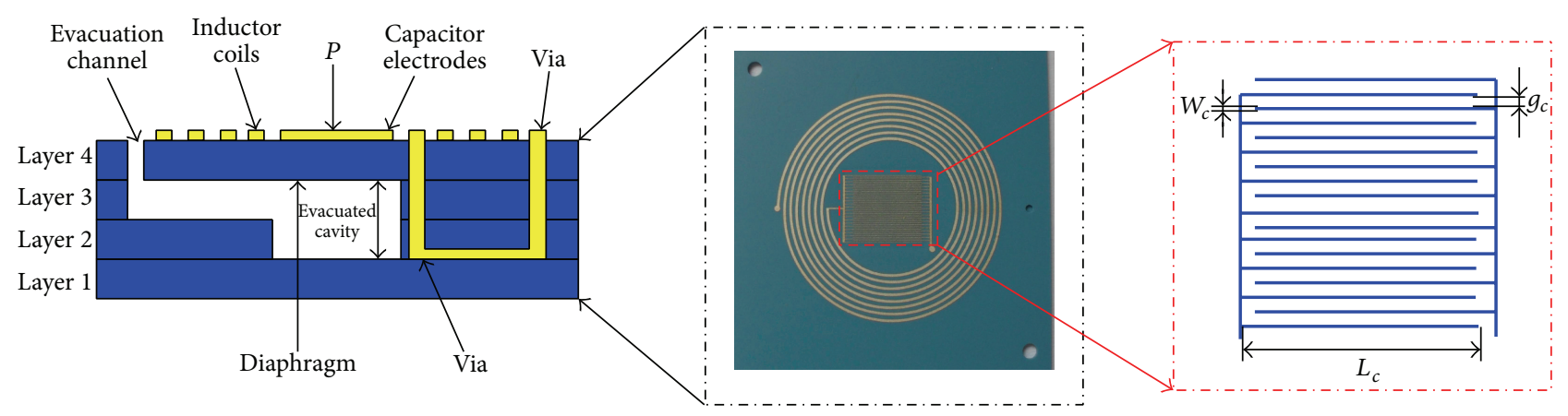

FIGURE 2: Schematic cross section.
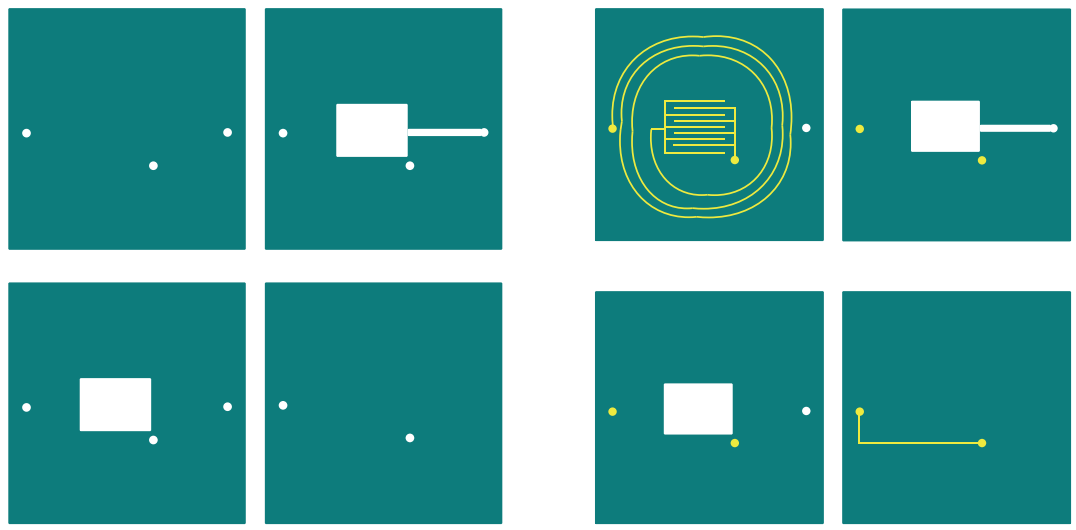

(1) Cut samples of green tape

(2) Via formation and pattern printing

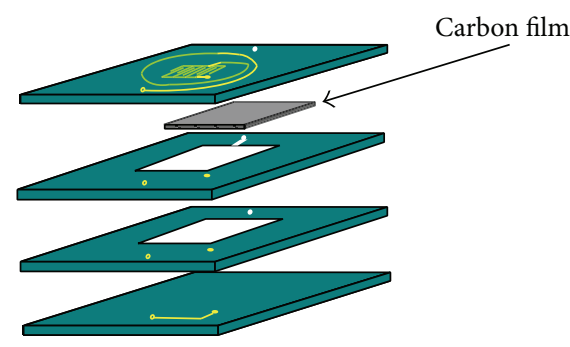

(3) Stacking

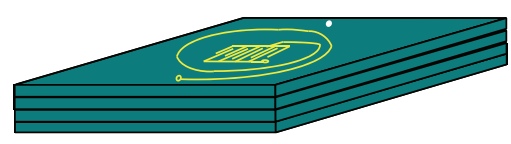

(4) Laminate sections
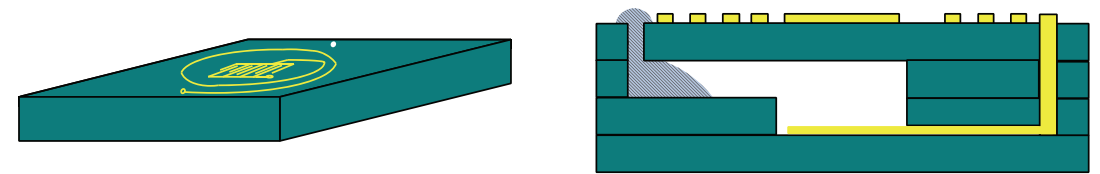

(5) Sinter sensor at high temperature

(6) Plugging evacuation channel with glass frit and melting to seal channel

FIGURE 3: Fabrication process of passive wireless sensor.

The sensor main production process is shown in Figure 3 as follows.

(1) Cut Samples of Green Tape. The punching document is loaded on computer. Then put the LTCC green tape on the punching machine. Samples are cut by laser.
Cavity structure and via will be formed, as shown in Figure 3, step 1.

(2) Via Formation and Pattern Printing. Inductor and capacitor were screen-printed, as illustrated in Figure 3, step 2. Via fill printing can ensure that the sensor circuit is connected. 
(3) Stacking. In the lamination process, it is necessary to use carbon film to fill the capacitance cavity in order to avoid transmogrification in sintering. The stacking temperature is $40^{\circ} \mathrm{C}$, as shown in Figure 3, step 3 .

(4) Lamination. The lamination adopts isostatic pressing in water. Put the sensors sample into the laminating machine in the isostatic hydraulic pressure of $15 \mathrm{MPa}$ for twenty minutes to make sensors have compact structure, as illustrated in Figure 3, step 4.

(5) Slice. After lamination, the sensors samples are to cut a square approximately $40 \mathrm{~mm}$ on a side prior to firing. This process can meet manufacture request.

(6) Sintering. The sensors samples are sintered in box furnace according to the set curve of temperature variation to harden the sample, as shown in Figure 3, step 5 .

(7) Seal. In Figure 3, step 3, the carbon film is used to fill the cavity, which forms the evacuation channel. It is necessary to use the glass particles to fill the evacuation channel. This is done by placing ESL4774-BCG glass frit (powdered glass) over the exit hole of the evacuation channel. The sample is placed in a vacuum tube furnace and heated at $540^{\circ} \mathrm{C}$ $\left(4^{\circ} \mathrm{C} \cdot \mathrm{min}^{-1}\right.$ ramp rate) and held for 10 minutes to melt the glass particles. The firing curve is shown in Figure 4. The powder average grain size is $20 \mu \mathrm{m}$, which is well suited for filling the evacuation channel, which can have a cross section of $800 \times 800 \mu \mathrm{m}$. The sensors are dealt with for 10 minutes at $540^{\circ} \mathrm{C}$ to melt the glass particles, as illustrated in Figure 3, step 6.

Air-tightness measurement is performed to guarantee the validity of the sealing process, which can be judged by the existence of bubbles. The sensor is placed in the high-pressure environment and is inflated for 2 minutes, and then it is placed into the water. The exhaust hole is observed through a magnifying glass if any bubble appears. After that, sensor without bubbles into the water is placed into the pressure tank to test pressure response. Theoretically, the frequency will decrease as the external pressure increases. In testing progress, the external pressure is 1 bar and is kept for at least $5 \mathrm{~min}$ and the frequency is recorded. If frequency is constant, it indicates that sensor has considerable air tightness.

\section{Experiments}

4.1. Pressure Measurement. The curves of magnitude and phase in the antenna port are shown in Figure 5. From Figure 5, it can be seen that the antenna and the sensor are coupled well. The frequency-pressure relationship of the sensor is shown in Figure 6. The frequency of the sensor decreases with the increase of the external pressure. The frequency-pressure characteristics present approximately linear, and the sensitivities of the interdigital capacitor pressure

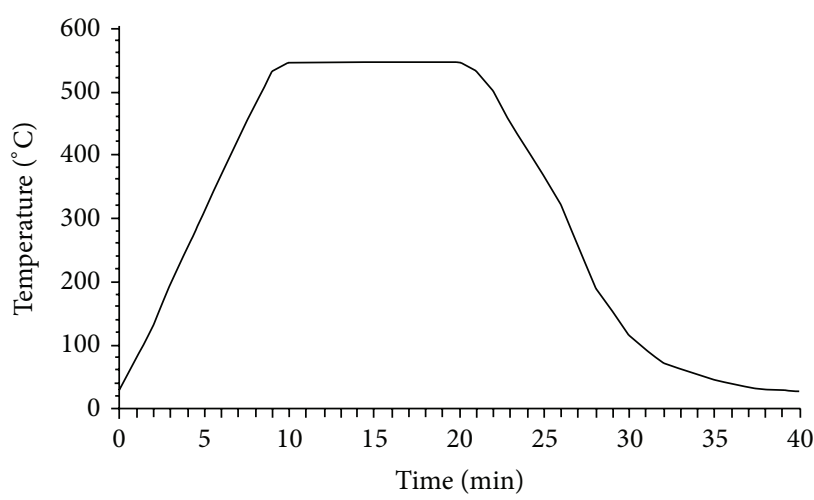

FIGURE 4: ESL-4774-BCG firing profile.

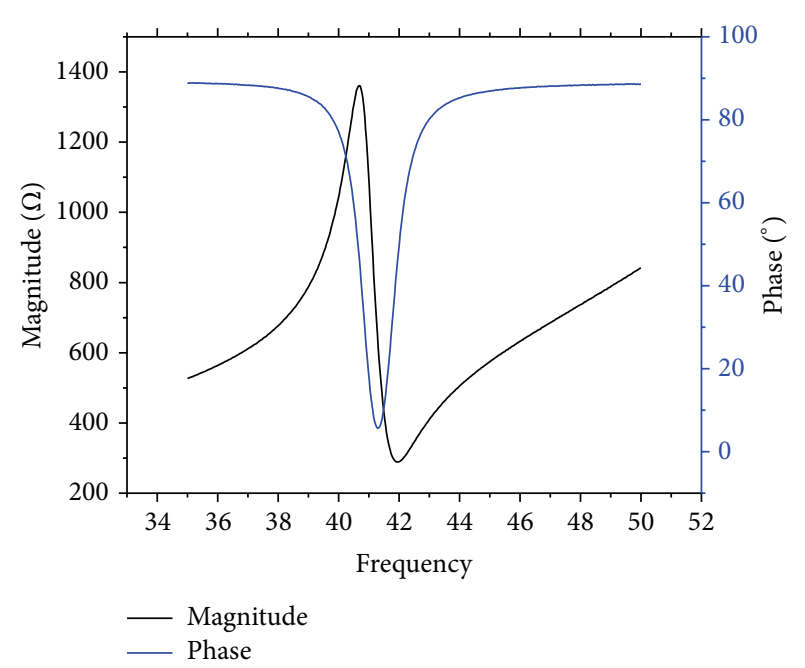

FIGURE 5: Measured magnitude and phase versus frequency for the pressure sensor.

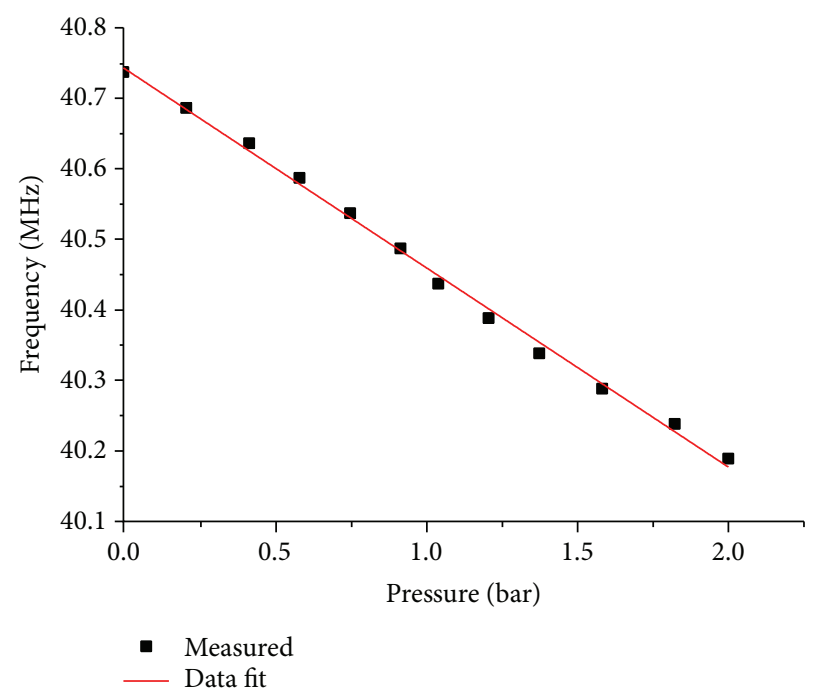

FIGURE 6: Resonant frequency versus pressure relationship. 


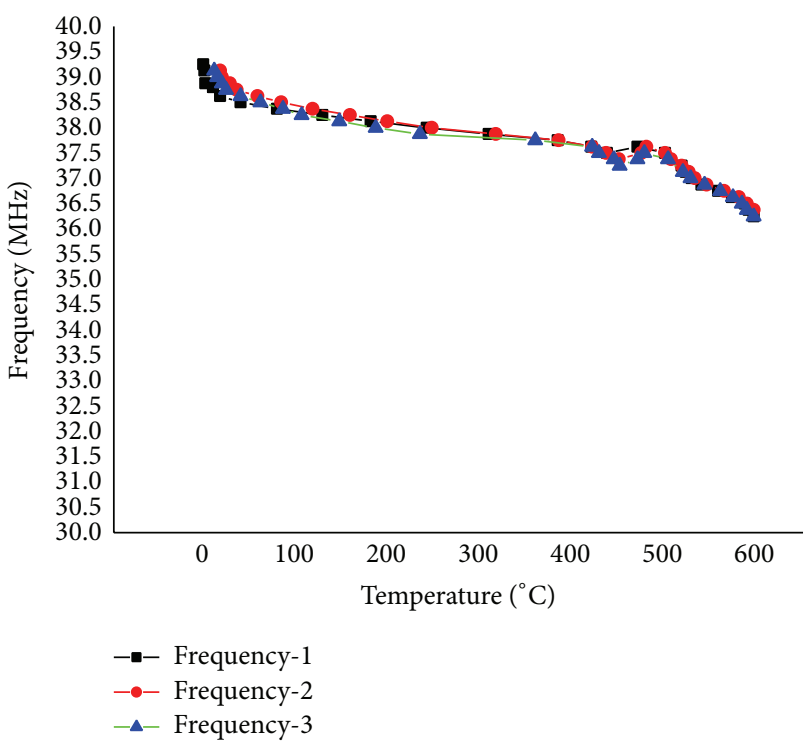

FIGURE 7: Resonant frequency versus temperature relationship.

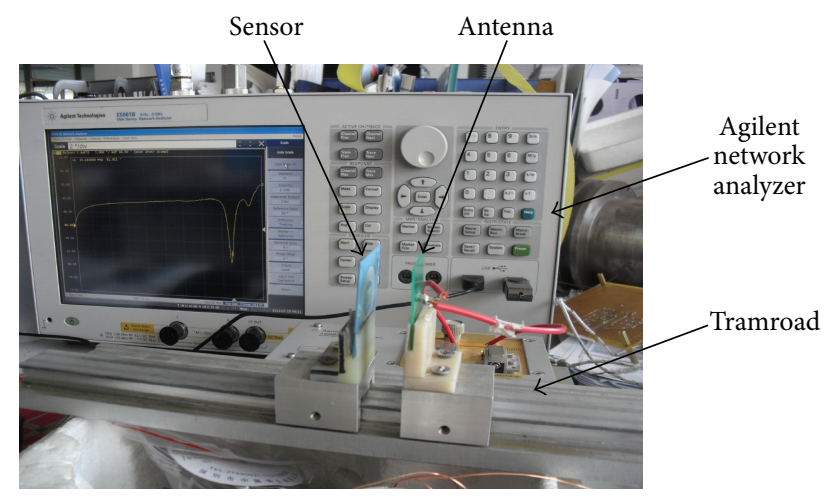

Figure 8: Photo of test system circuit.

sensor are approximately $273.95 \mathrm{kHz} /$ bar with a readout distance of $25 \mathrm{~mm}$. In the paper, the inductor windings of the sensor are circular; the quality factor $Q$ of circular inductor is better than square inductor. The maximum test pressure limited by the equipment is 2 bar. Otherwise, the pressure sensitivity could be optimized by enlarging the dimension of the evacuated cavity to improve the ratio of load-deflection.

4.2. Temperature Measurement. In order to verify the hightemperature performance of sensor, a high-temperature experiment is required. We use tungsten filament as the test antenna. The test temperature is $25-600^{\circ} \mathrm{C}$. Heating process is as follows: we measured the sensor from room temperature to $600^{\circ} \mathrm{C}$ and then at $600^{\circ} \mathrm{C}$ for $30 \mathrm{~min}$. The frequency-pressure relationship of the sensor at room temperature is shown in Figure 7.

These results show that the resonant frequency of sensor decreases with the increase of temperature, which coincides with the fact that the relative dielectric constant $\varepsilon_{r}$ of the LTCC green tape is changing with temperature. Finally, the heat stability test is carried out. Sensors left at $600^{\circ} \mathrm{C}$ over a

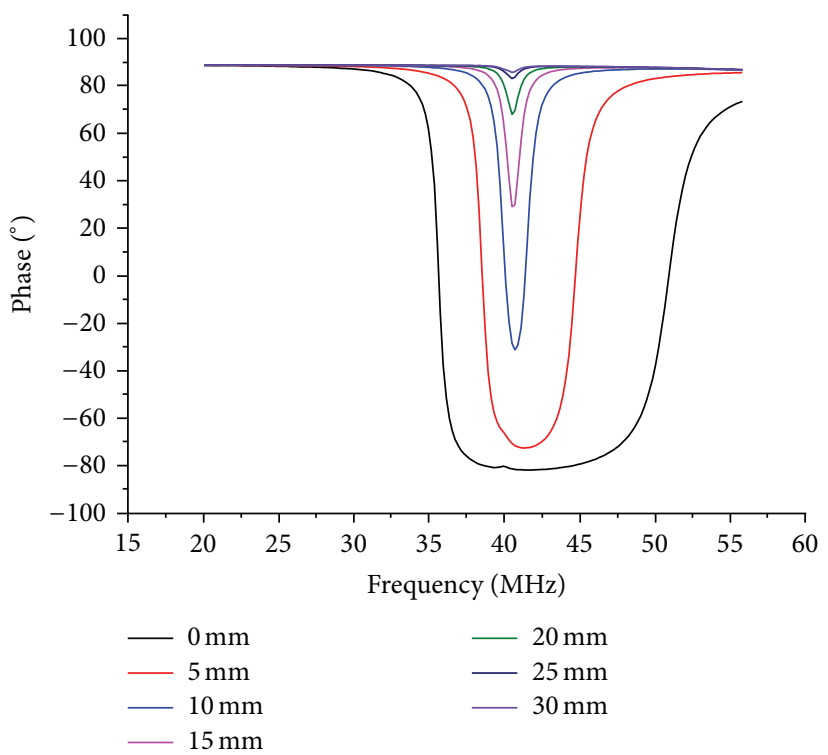

FIGURE 9: Coupling distance measurement.

$12 \mathrm{~h}$ period did not exhibit a change in resonant frequency or pressure sensitivity nor did they exhibit hysteresis.

4.3. Readout Distance Measurement. In order to test the coupling performance of the sensor, we set up as shown in Figure 8 test platform. The sensor and the antenna are placed in parallel. Figure 9 shows the coupling relationship between distance and phase.

The distance between sensor and antenna is $0 \mathrm{~mm}$; the peak of the phase is flat. The distance between sensor and antenna is $10 \sim 20 \mathrm{~mm}$; the phase has a very sharp peak. The distance between sensor and antenna is $30 \mathrm{~mm}$; coupling waveform is less obvious. The distance between sensor and antenna is far-forth than $30 \mathrm{~mm}$; sensor can no longer be coupled with the antenna.

\section{Conclusions}

In this paper, a passive contactless pressure sensor was fabricated using LTCC technology. The sensor detected pressure variations by using changes in its resonant frequency. The test results show that the resonant frequency of sensor decreased with the increasing of external pressure. The sensitivity of the resonant frequency change is $273.95 \mathrm{KHz} /$ bar. Experimental results showed that the data of external pressure can be detected at $600^{\circ} \mathrm{C}$ with a distance of $25 \mathrm{~mm}$ and also that the sensor has higher stability at this temperature. Furthermore, the proposed sensors can be used to measure pressure at high temperature $\left(400-600^{\circ} \mathrm{C}\right)$.

\section{Conflict of Interests}

The authors declare no conflict of interests. 


\section{Acknowledgments}

This work was supported by the Program for the Outstanding Innovative Teams of Higher Learning Institutions of Shanxi, the National Science Fund for Distinguished Young Scholars (no. 51225504), and the Visiting Scholar Fund from National Key Laboratory of Fundamental Science of Micro/NanoDevice and System Technology of Chongqing University (no. 2013MS03).

\section{References}

[1] M. A. Fonseca, J. M. English, M. Von Arx, and M. G. Allen, "Wireless micromachined ceramic pressure sensor for hightemperature applications," Journal of Microelectromechanical Systems, vol. 11, no. 4, pp. 337-343, 2002.

[2] G. W. Hunter, P. G. Neudeck, R. S. Okojie, G. M. Beheim, J. A. Powell, and L. Chen, "An overview of high-temperature electronics and sensor development at NASA Glenn Research Center," Journal of Turbomachinery, vol. 125, no. 4, pp. 658-664, 2003.

[3] W. A. Vitriol and J. I. Steinberg, "Development of a low temperature, cofired multilayer ceramic technology," International Journal of Microcircuits and Electronic Packaging, vol. 6, no. 1, pp. 593-598, 1983.

[4] J. M. English and M. G. Allen, "Wireless micromachined ceramic pressure sensors," in Proceedings of the 12th IEEE International Conference on Micro Electro Mechanical Systems (MEMS '99), pp. 511-516, January 1999.

[5] R. Nopper, R. Niekrawietz, and L. Reindl, "Wireless readout of passive LC sensors," IEEE Transactions on Instrumentation and Measurement, vol. 59, no. 9, pp. 2450-2457, 2010.

[6] J. C. Butler, A. J. Vigliotti, F. W. Verdi, and S. M. Walsh, "Wireless, passive, resonant-circuit, inductively coupled, inductive strain sensor," Sensors and Actuators, A: Physical, vol. 102, no. 1-2, pp. 61-66, 2002.

[7] E. L. Tan, W. N. Ng, and R. Y. Shao, "A wireless, passive sensor for quantifying packaged food qulity," IEEE Sensors Journal, vol. 11, pp. 1747-1756, 2007.

[8] L. E. Khoong, Y. M. Tan, and Y. C. Lam, "Overview on fabrication of three-dimensional structures in multi-layer ceramic substrate," Journal of the European Ceramic Society, vol. 30, no. 10, pp. 1973-1987, 2010.

[9] C. Gehin, C. Barthod, and Y. Teisseyre, "Design and characterisation of a new force resonant sensor," Sensors and Actuators, A: Physical, vol. 84, no. 1, pp. 65-69, 2000.

[10] H. Birol, T. Maeder, and P. Ryser, "Processing of graphite-based sacrificial layer for microfabrication of low temperature co-fired ceramics (LTCC)," Sensors and Actuators A: Physical, vol. 130131, pp. 560-567, 2006.

[11] M. A. Fonseca, Polymer/ceramic wireless MEMS pressure sensors for harsh environments: high temperature and biomedical applications, [Dissertation Abstracts International], 2007, 68-12(B): 8247. 

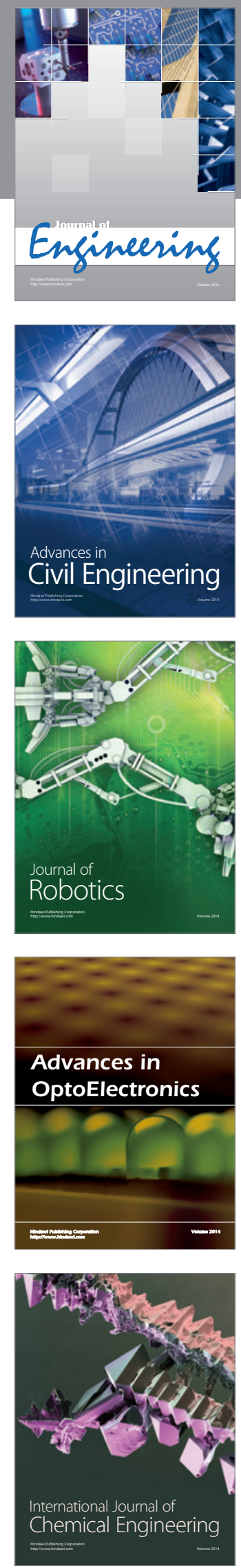

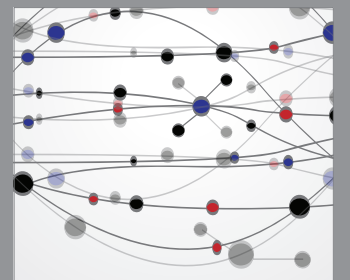

The Scientific World Journal
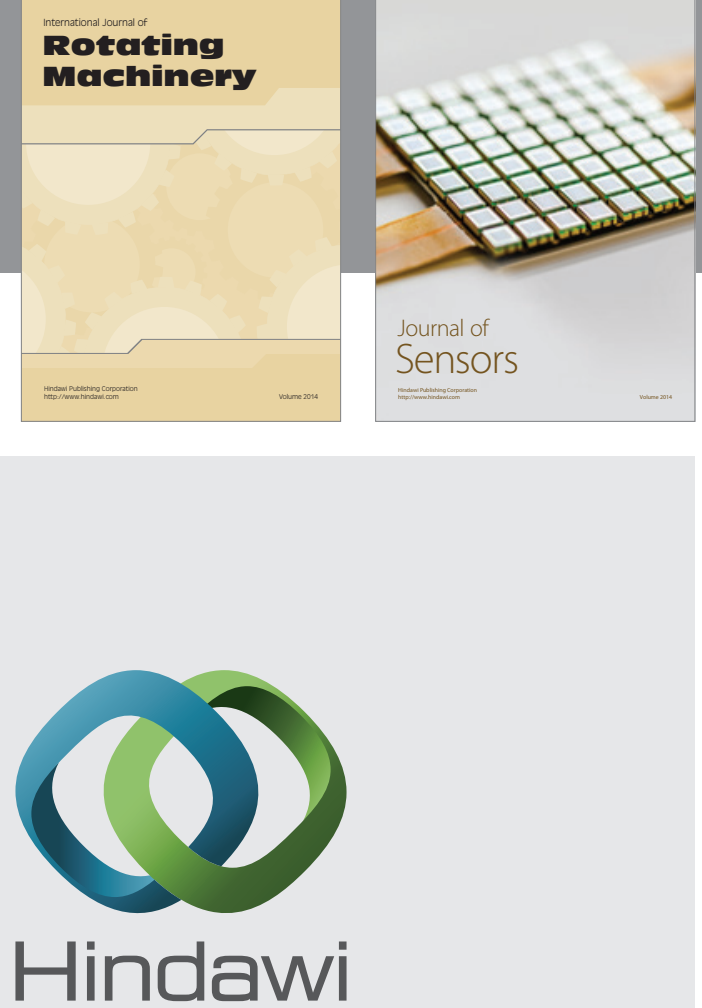

Submit your manuscripts at http://www.hindawi.com
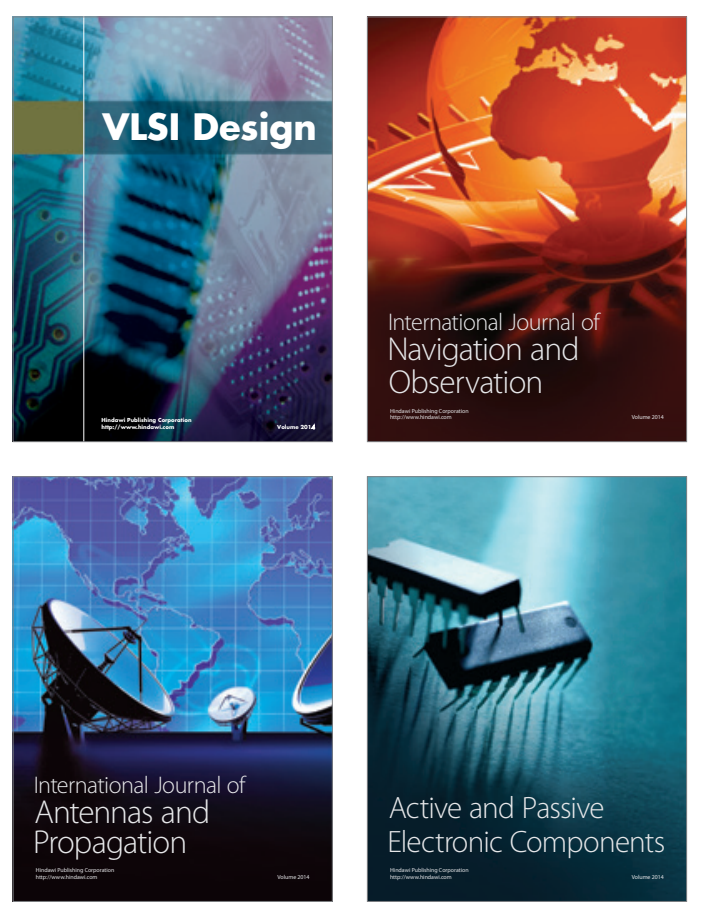
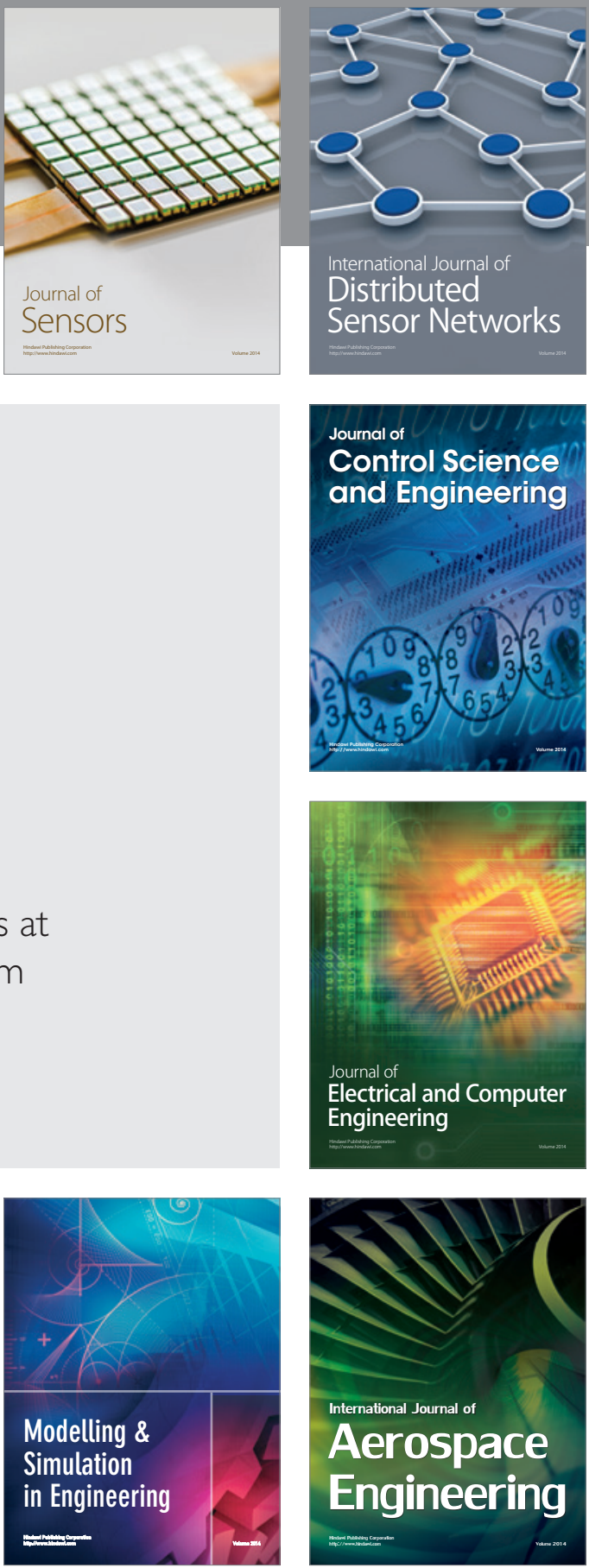

Journal of

Control Science

and Engineering
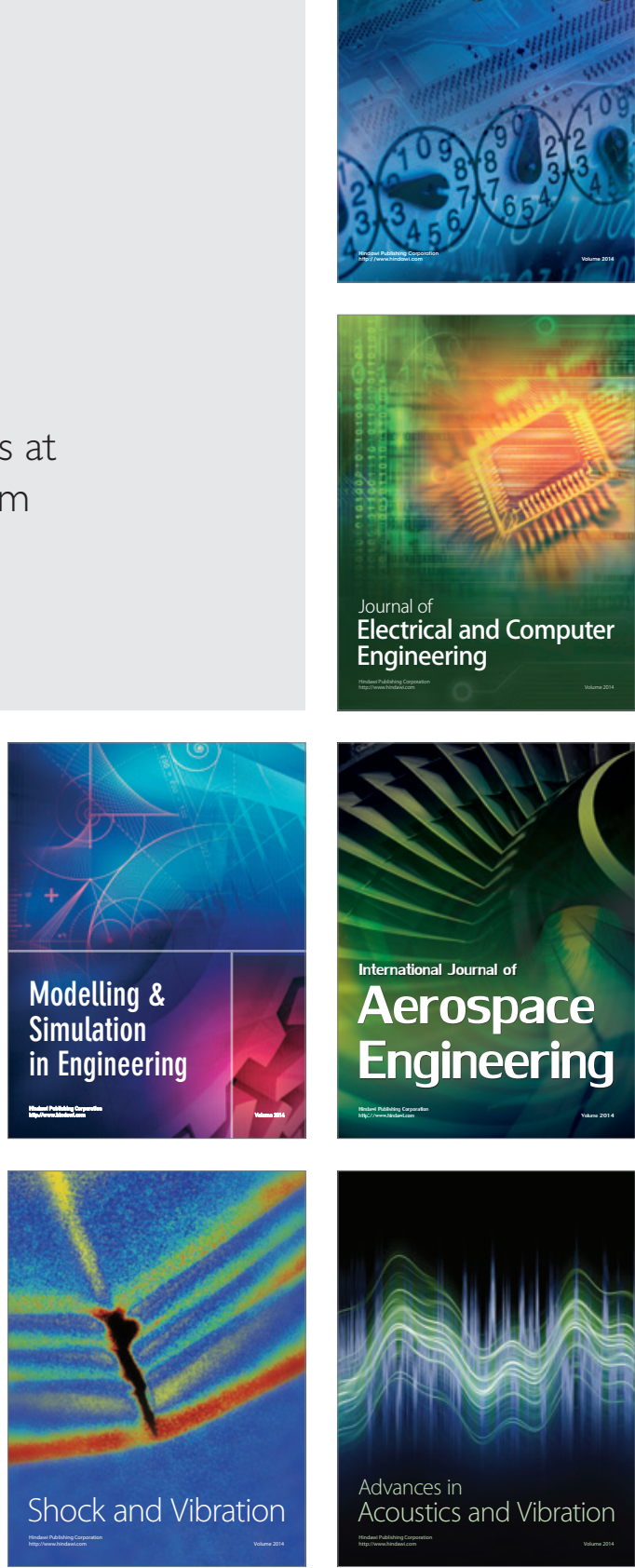